\title{
The Research of AGV Guidance Systems Based on RFID Positioning
}

\author{
Shixian Zeng ${ }^{1, a}$, Rui Xue ${ }^{1, b}$, Yang Wang ${ }^{1, c}$, Dengwei Tian ${ }^{1, d}$ \\ ${ }^{1}$ Qingdao Huanghai University, China \\ a zsx113@126.com, bxuerui126@126.com, 'c1149643940@qq.com, ${ }^{\text {d } 598369484 @ q q . c o m ~}$
}

Keywords: Storage guided vehicle system, RFID technology, Electronic tags.

\begin{abstract}
According to the characteristics of the transport system, RFID technology is integrated in the AGV automatic guided vehicle system in the warehousing. The node RFID oriented method is chosen and the RFID note positioning function is used to realize more types and over horizon identification loading, handling and automatic storage function that improves the system flexibility through the design of tag oriented system.
\end{abstract}

\section{Introduction}

With the development of the AGV, it has become an integral part of the logistics and distribution system of logistics equipment, which can play a strong function in production and transport, logistics and distribution systems and therefore this device can be further developed and applied [1]. AGV (Automated Guided Vehicle) is a flexible and intelligent as one of an efficient load handling equipment, through the built-in automatic guidance system can be automatically loaded in the case of manual handling of non-intervention. It works by using the communication between the AGV and the host server, thus enabling the system to accept the task instructions issued by the host computer, the execution so that equipment running from the origin to the end, the use of the characteristics of the device itself to complete the assigned agency goods transportation and handling[2].Paper, production through automation, intelligent transportation research, the use of RFID technology[3][4], the entire handling system overall design and planning, thereby reducing the use of human and material resources, and the working efficiency of the system is increase.

\section{Guidance System Composition}

System Overview.Herein storage guide system consists of a power supply system, the vehicle body, a drive system, wireless communication device, the control center, the positioning module and other components. AGV PC to receive a corresponding path planning, through execution of the corresponding command control center, enabling the efficient movement of the system. The three functional objectives are completed in design, control and implementing agencies. The guidance system is mainly composed of the AGV host computer control system, the vehicle control systems, sensor module, motion systems, RFID readers, tags and wireless communication module cargo and other components. System hierarchy is shown in Figure 1.

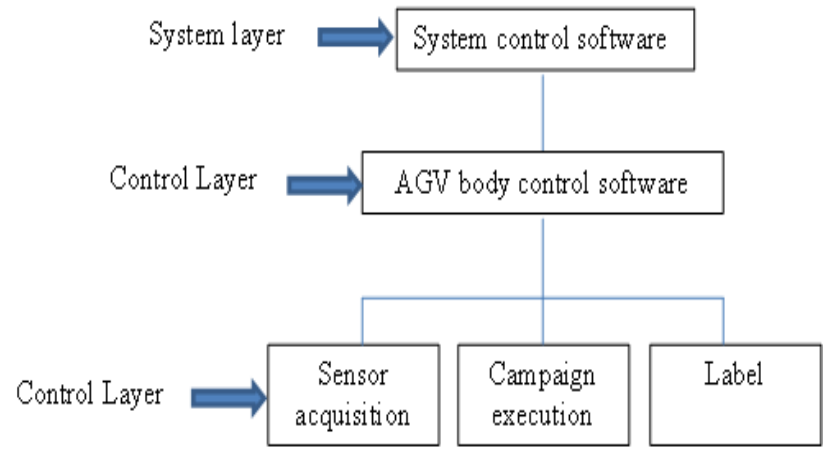

Figure 1 Hierarchy of a schematic diagram of the system 
Hardware Components. The system hardware consists of many different sub-modules, specifically for wireless local area networks, computer systems, motor, electronic code disk, automatic guided vehicle power supply, various sensors, control center. Composed of hardware modules is shown in Figure 2.

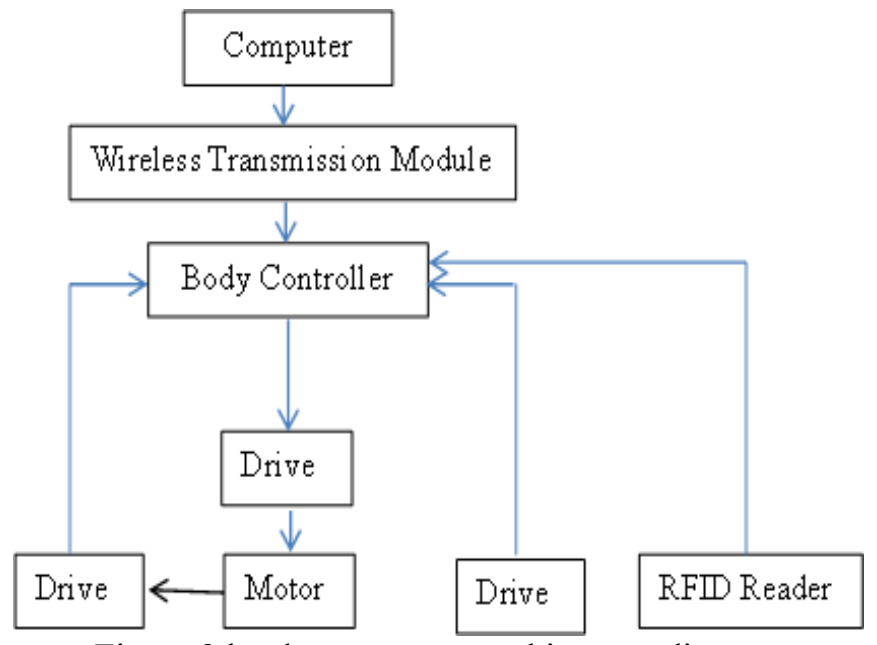

Figure 2 hardware system architecture diagram

The entire hardware system is the most important device for the computer, which uses a standard server, equipped with the software system, whose duty is to give instructions AGV system. Information in this system the top intermediate layer and the transfer of information over the wireless local area network, different wireless local area network comprises a different communication protocol, which requires communication in the corresponding communication protocol. Work DC motor play a role in the motion control system, the driver is determined primarily by the $\mathrm{H}$-bridge PWM wave to control speed. AGV is responsible for driving the encoder speed feedback, the feedback that the running speed and distance information systems, two DC motors makes the AGV's attitude is adjusted by adjusting the speed. The design of the sensor system uses paper are: avoidance of ultrasonic sensors, infrared sensors, these sensors collect information about the environment is used to control the AGV sport, attitude adjustment data basis ${ }^{[5]}$.

Software Components. Storage automated guided vehicle system software and software from the top of the middle layer of the MCU system PC control software. AGV core software components of the system is the top of the PC control system, which has a remote control command for automatic guided vehicles, issued function commands and tasks. While AGV and storage corresponding status information is real-time custody, the AGV path is planned by PC control system ${ }^{[6]}$.

AGV main body of software running on the microcontroller to control the implementation of its responsibilities for the information of the PC control system integration translation, thus providing control instructions for each actuator, and feedback to the PC control software to collect relevant information ${ }^{[7]}$.

\section{RFID Integrated Design and AGV}

RFID Technology Principle and Composition.RFID (Radio Frequency Identification) refers to the radio frequency identification, is a more advanced wireless identification technology. The technology RFID reader for RFID-related information on the RFID read, thus completing the application and implementation of the guidance system, through user-defined label information, allowing the system to achieve efficient guiding effect.

RFID system includes RFID readers and RFID electronic tags usually ${ }^{[8]}$. Target information is required by the system, the reader reads the electronic tag after the target information, the information corresponding data processing. Process required by the RFID reader connected to the computer serial port corresponding to the data obtained by the feedback to the operator via a computer interface, so, RFID system is a necessary part with RFID technology is matched. 
RFID Systems Integration and AGV.By integrating RFID systems and AGV, such that the guide effect of the system is improved. Figure 3 shows a schematic diagram of an integrated system composed of communication in the serial RFID reader and AGV control system implemented in the corresponding communication protocol to transfer the collected data reader to AGV control systems, real-time control system to upload data to PC, PC to analyze data in order to achieve the integration of the whole process of AGV and RFID systems.

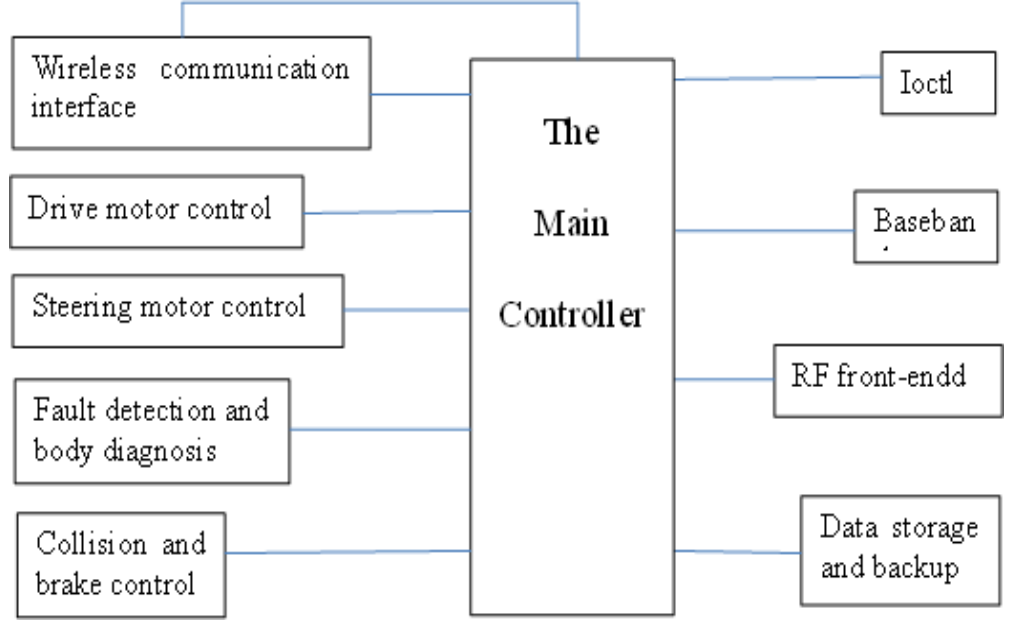

Figure3 Schematic of system integration

Integrated System Driven Design. LPC2210 microprocessor-driven control system is an integrated control unit with the core, which is mainly used for each interface data processing, data storage and connection system, the system control functions. The control unit includes the following major components: the microcontroller LPC2210, Serial and Modem port, debug interface. Si4133 is the system used in drive control chip, which consists mainly of startup programs: detect lock bit function Si4133_Locked, Si4133_SendData function to send data to the Si4133. The flowchart Si4133_Locked function is shown in Figure 4.

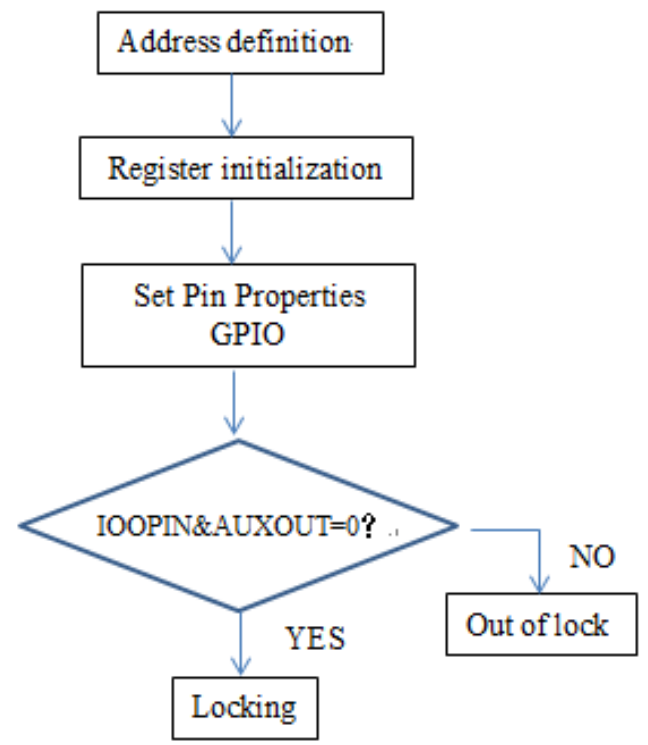

Figure 4 Si4133_Locked function flowchart

\section{RFID Tag-oriented Design}

RFID Standards.Currently, more authoritative electronic tag has two major research institutions, which can represent an electronic tag force to the development of standards. One was established in 1999 and headquartered in Massachusetts Institute of Technology, and the other in the afternoon in March 2003 was established in Japan, the two centers introduced standardized specifications are some differences. 
In China, RFID technology has been the use and development of industry standards or only in some independent research and development of various RFID tag products. In terms of capacity and information format, the technology contains inconsistencies and incompatibilities. Therefore, China needs some independent research as soon as the appropriate standard for my label standard.

Front-end Circuit.Systems electronic tags by the RFID circuit and antenna, divided into two types: active and passive type. Passive tags do not need to work in the power, low cost, high reliability teach; active tags require a power supply work process, when not energized in "standby" state, when excited by the reader into the normal working communication state. The study found that active tags for location and information processing advantage, therefore, greater stability. Front-end circuitry is shown in Figure 5.

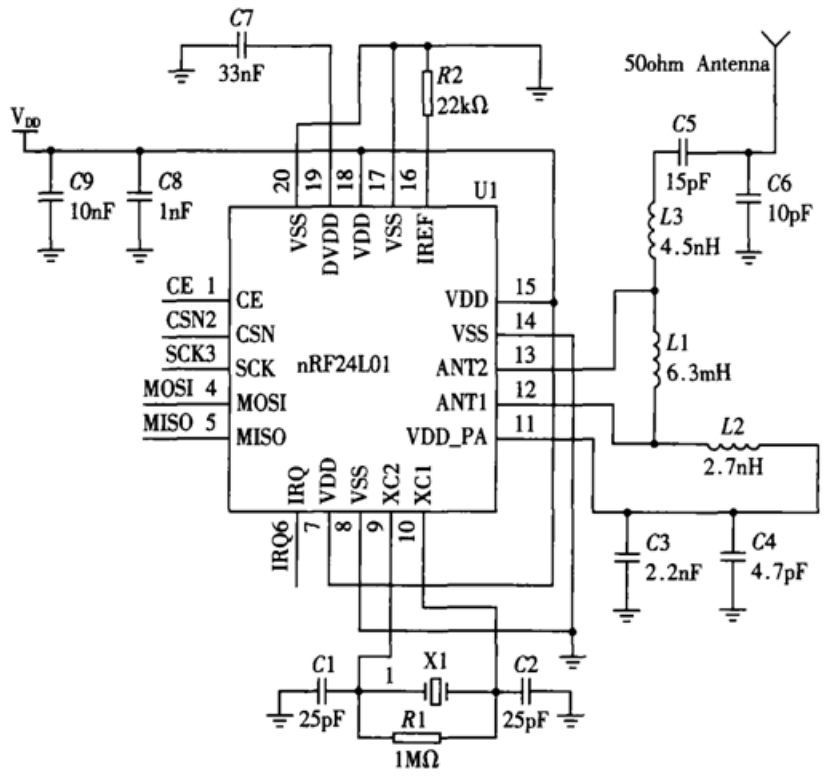

Figure 5 Front-end circuitry

Label Software Design .For the reception and transmission, RF transceiver chip function data play a key role, in which the software configuration register can achieve a lot of features, including: a transceiver frequency, receiving address, CRC checksum length, wireless receive mode. Packet consists of a preamble, address, packet label, valid data and CRC check of five parts. Coordination of work between the hardware for efficient transmission and reception of data packets have a great impact, for example, the preamble information can be done automatically sending and receiving under hardware control. Data packets are labels, can be effectively prevented repeatedly received data controller.

When the system receives a command packet (address match, CRC correct), the received data is stored in the status register RX_FIFO. Position level control IRQ status register is valid. When the instruction so that the system automatically valid, acceptable response signal will be returned. CE micro-program controller is low, the control system will enter a low-power mode, this time, the system will carry out, such as the instruction fetch and store at low current. Transmitting and receiving the flowchart described herein tag instruction operates in FIG 6 and 7, respectively, the micro-controller to perform read and write operations in the process, which accurately processing RFID work-related information play a key role. 


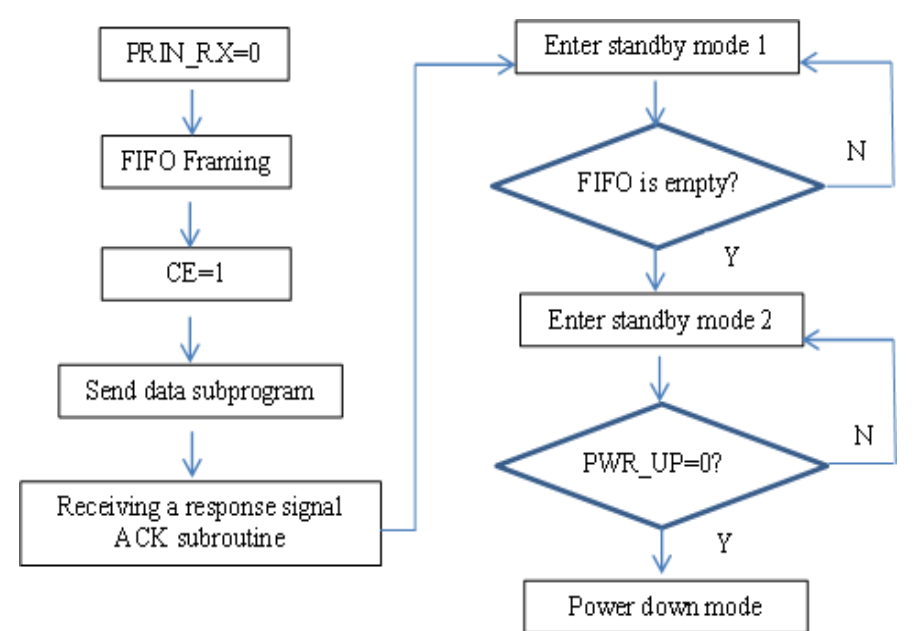

Figure 6 Send schematic label instructions

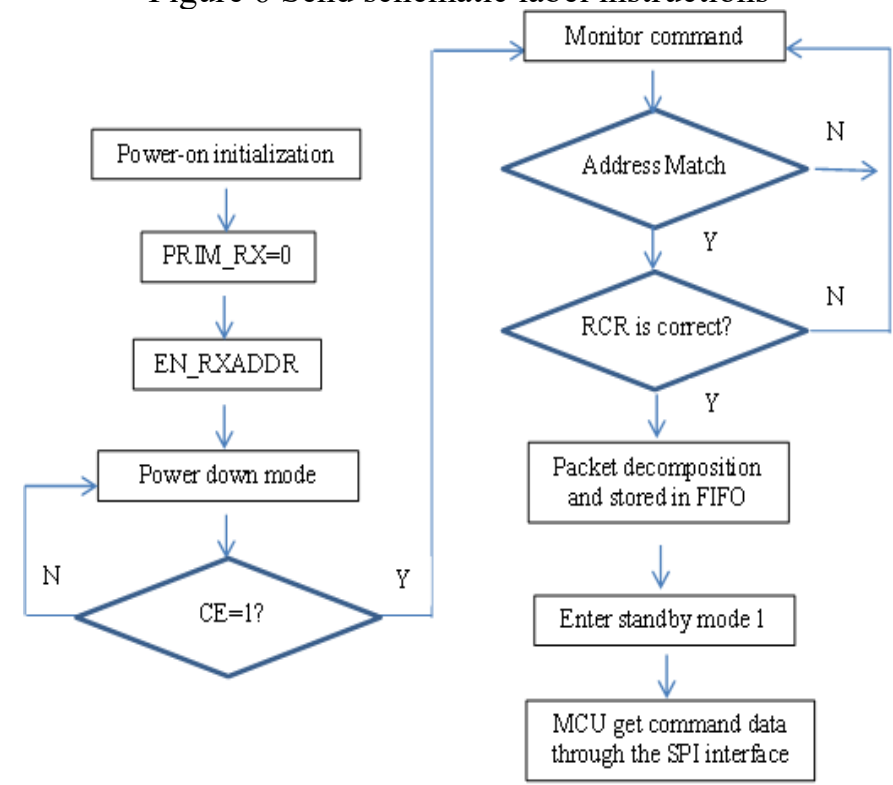

Figure 7 label instructions to receive schematic

Determined command transmission effect mainly by accepting the response signal, if the system receives a corresponding response signal, the data begins to delete TX FIFO, this time describing the instructions transfer was successful; on the contrary, the data has been transmitted, the automatic retransmission valid state. Data transfer instruction rely automatic retransmission counter counts, when the count value exceeds the maximum limit register is set, the high status register MAX_RT position, this time, IRQ effective information transmission process will produce MAX_RT interruption, while PLOS_CNT lost count of the counter is incremented, the command is sent after entering standby mode. RFID tag reader after receiving information, send "PWR_UP $=0$ ", this time, the electronic tag enters power-down mode, the entire process efficient and stable transmission of data to complete the information.

System is mainly used for communication test. The system reset, the computer's COM port DART serial communication module for the pre-configured with the same parameters baud rate, set the serial port baud rate of $9600 \mathrm{bps}$ logistics tracking system in the Device Manager PC software and connected to the appropriate COM port. If the RFID reader has a response that communication is successful, if the communication quality is poor, need to loop tuning parameters, and ultimately communicate.

Through simulation and testing shows that, RFID and AGV systems in acquisition and integration of data transfer rates faster feedback system waits a short time, greatly improving the operational efficiency of the system. Thanks to intelligent features designed AGV system to greatly reduce labor requirements, and further improve the accuracy for the transport control for greater help to improve economic efficiency. 


\section{Summary}

This paper discusses and designed a combination of RFID and AGV integrated systems, this design can be used in production and transport. According AGV design features RFID and integrated systems, while oriented technology combined with RFID tags, enabling multi-target production, BVR recognition and loading, handling and automatic storage function, increase the flexibility of the system.

Using a monolithic integrated RF front-end, simplifying the application system, the stability and reliability of wireless systems is enhanced, while making the research and development easier, reducing the cost of the system, the whole system of low power consumption, small size, easy to expand, rebuild convenient, high reliability, suitable for efficient production.

\section{References}

[1] Lifang Wang, Guoliang Yuan, Feng Guo. RFID Warehouse Management System of [J] Science Technology and Engineering, 2011.15: 3567-3570, in Chinese.

[2] Pan Zhang. Design and implementation of materials warehouse management system based on RFID technology [D]. Electronic Science and Technology University,2013, in Chinese.

[3] Mengru Cao. The research and application of warehouse management system based on RFID [D].Anhui Agricultural University.2013, in Chinese.

[4] Huagui Deng. Design and Implementation of supermarkets warehouse management system based on RFID [D].Jilin University, 2014, in Chinese.

[5] Shaoping Lu. AGV positioning and guiding research based on RFID [D]. Shandong University, 2011, in Chinese.

[6] Yongding Wang, Jiapeng Yang. RFID and AGV systems integration and application in the distribution center [J]. Computer Systems Applications, 2011.11: 131-134, in Chinese.

[7] Beisi Zhang Chen, Automatic guided vehicle (AGV) Summary of development [J]. Chinese manufacturing information, 2010.01: 53-59, in Chinese.

[8] Lei Lei, Jisheng Wu, Fangyu Chen. The Research of Logistics automation warehouse positioning method based on RFID [J]. Logistics Technology, 2012.03: 103-105 + 148, in Chinese. 